\title{
Photoacoustic imaging with a multi-view Fabry-Pérot scanner
}

\author{
R. Ellwood ${ }^{1}$, Felix Lucka ${ }^{2}$, E.Z. Zhang ${ }^{1}$, P.C. Beard ${ }^{1}$, and B.T. Cox ${ }^{1}$ \\ ${ }^{1}$ Department of Medical Physics and Biomedical Engineering, University College London, \\ Gower Street, WC1E 6BT, UK \\ ${ }^{2}$ Department of Computer Science, University College London, Gower Street, WC1E 6BT, UK
}

\begin{abstract}
Planar Fabry-Pérot (FP) ultrasound sensor arrays have been used to produce in-vivo photoacoustic images of high quality due to their broad detection bandwidth, small element size, and dense spatial sampling. However like all planar arrays, FP sensors suffer from the limited view problem. Here, a multi-angle FP sensor system is described that mitigates the partial view effects of a planar FP sensor while retaining its detection advantages. The possibility of improving data acquisition speed through the use of sub-sampling techniques is also explored. The capabilities of the system are demonstrated with 3D images of pre-clinical targets
\end{abstract}

\section{Introduction}

Thin-film Fabry-Pérot (FP) interferometric ultrasound sensors have been used to produce photoacoustic images of high quality[1], [2]. For simplicity of manufacture, FP sensors are produced in a planar form. As with all planar detectors, images produced with data from a single FP sensor array suffer from limited view artifacts[3], [4]. In a previous work [5]-[7] it has been shown that the use of two orthogonal FP sensor planes can produce images with reduced artifacts and sharper details in ex-vivo conditions. Here the use of multiple additional planes to further increase the solid angle subtended by the array is explored.

Multiple sensor planes were synthesised by rotating the imaged target relative to the sensor plane. Whilst rotating the target is not a novel way of dealing with the limited-view problem [8]-[12], it has not previously been implemented using a FP sensor, and allows different arrangements to be tested at this proof-of-principle stage. For example, the positions of the multiple planes can be altered by changing the distance between the centre of rotation and sensor plane, by changing the size of the region of the sensor that is interrogated, by changing the size of the angle between subsequent sensor positions, and by changing the overall number of sensor positions.

\section{System design}

The system, shown in Fig. 1, consisted of a planar Fabry-Pérot thin film interferometer [1], mounted vertically, and a custom interrogation system mounted horizontally relative to the sensor. The sample was held directly in front of the sensor in a custom sample holder, which was rotated by a stepper motor (Sanyo Denki 103H5208$0440)$. Excitation light, from a Quantel Ultra $(\lambda=1064 \mathrm{~nm}, 8 \mathrm{~ns}$ pulse width, $20 \mathrm{~Hz}$ repetition rate), was introduced into the volume in backward mode through the sensor.

Photons Plus Ultrasound: Imaging and Sensing 2017, edited by Alexander A. Oraevsky, Lihong V. Wang, Proc. of SPIE Vol. 10064, 100641F · (c) 2017 SPIE · CCC code: 1605-7422/17/\$18 · doi: 10.1117/12.2252728 


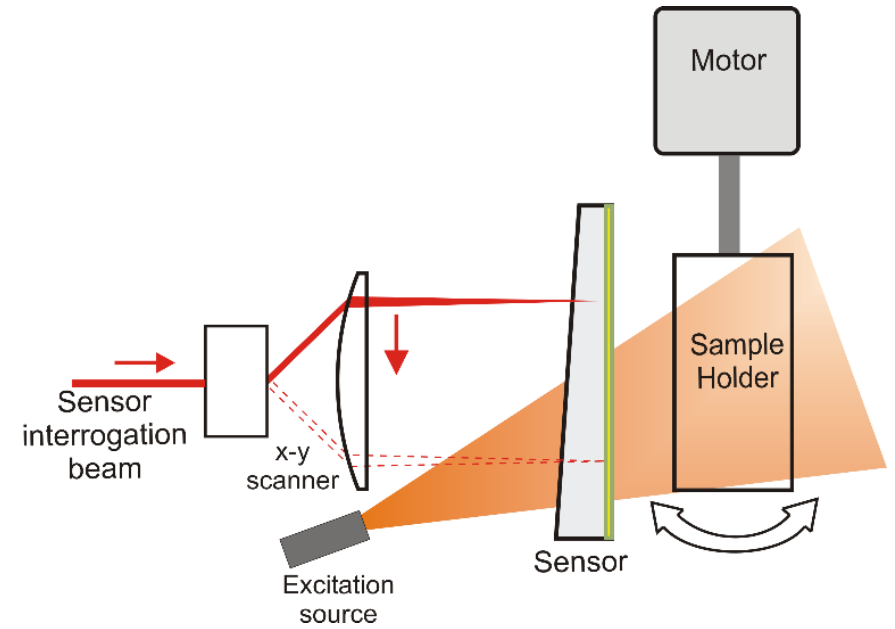

Figure 1 System diagram. Standard Fabry-Pérot(FP) sensor mounted vertically, interrogated by a custom interrogation system. The sample is held in front of the sensor in a custom mount. The sample mount is rotated by a stepper motor. Excitation light is introduced in backward mode through the sensor. The water tray used to acoustically couple the sample to the sensor is not shown.

\section{Registration procedure}

In order to register all of the planes' local coordinate systems to a single global coordinate system, a registration procedure was developed. A registration phantom consisting of three separate $17 \mu \mathrm{m}$ polymer strands was photoacoustically imaged from each sensor position. In this case six angles, each $45^{\circ}$ apart, were used to give a total angular aperture of $225^{\circ}$. The separate images for each plane/angle were reconstructed using time reversal[13], [14], and for each a point cloud was generated by thresholding the voxels based on their image intensities (Fig. 2(a)). A rigid point set registration [15] was then used to align the point clouds and thereby register each plane to the universal coordinate system. The six planes registered to a single universal coordinate system can be seen in Fig. 2(b).

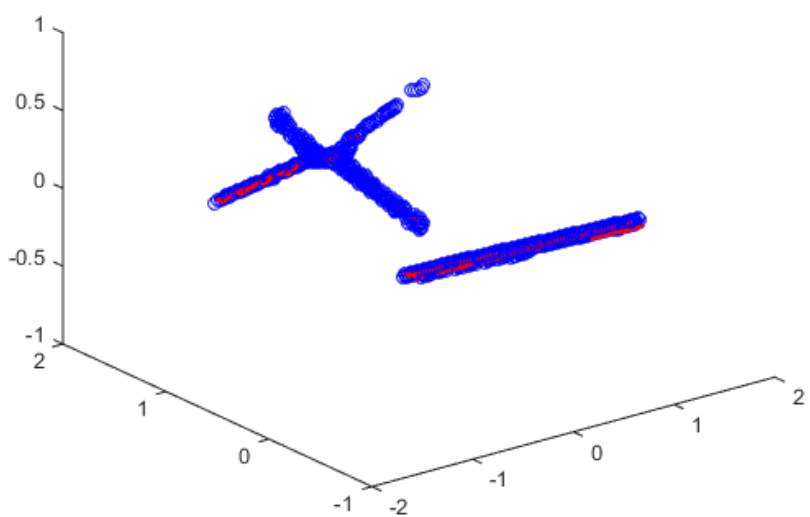

(a)

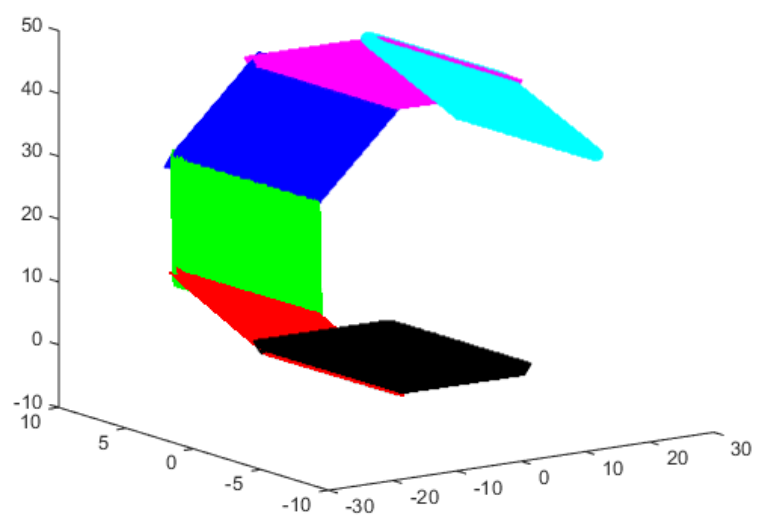

(b)

Figure 2 (a) demonstration of two point clouds generated from two different planar positions having been registered using the rigid point registration. (b) Position of 6 planes registered to global co-ordinate system.

\section{Reconstruction}

An iterative time-reversal image reconstruction algorithm[16], [17] was used to obtain a single photoacoustic image from the combined data from all sensor planes. An iterative technique has the benefit of allowing the dual physically-justified constraints of zero initial acoustic particle velocity and non-negative initial acoustic pressure to be enforced. This improves the quality of the image by, for example, reducing the level of artifact, compared to a linear sum of the individual images. Fig. 3 demonstrates this with two reconstructions of the registration phantom obtained with different algorithms. In Fig. 3(a) the image is the sum of the images obtained for each 
individual plane, whereas in Fig. 3(b) the image was iteratively reconstructed using the data from all planes simultaneously. In Fig. 3(a) negative artifacts are clearly present, however in Fig. 3(b) these are not present due to the improved reconstruction technique. Furthermore, although it is not visible, as both images are normalised to their respective maximum values, the iterated reconstruction, Fig. 3(b) has features with larger magnitudes than the summed image. The acoustic forward model used in the iterative time-reversal image reconstruction scheme was a k-space pseudospectral time-domain propagation model, k-Wave [18].
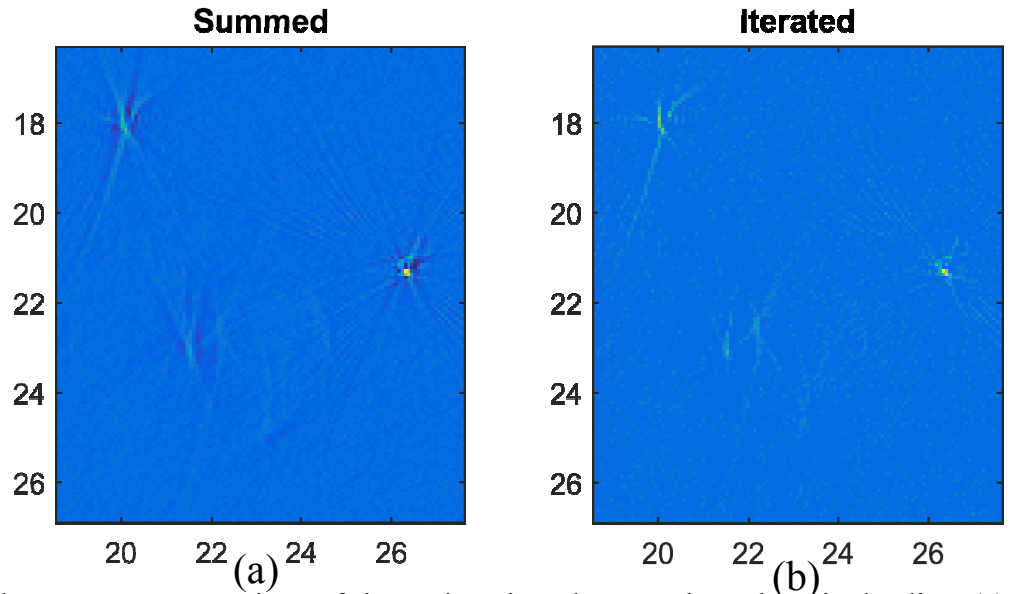

Figure 3 comparison between reconstructions of the registration phantom through a single slice. (a) Summed independent reconstructions for each plane. (b) Iterated time reversal using data from combined sensor geometry. Both reconstructions are plotted on a normalised scale. The intensity of the iterated features is double that of the summed reconstruction. Negative artifacts are present obvious in the summed reconstruction.

\section{Experimental results}

To demonstrate the system's ability to photoacoustically image complex structures over a large region, two phantoms are imaged, a skeletal leaf phantom and tube phantom tied in a knot.

\section{Skeletal leaf phantom}

The skeletal leaf phantom has fine structure running at several different angles, making it challenging to image with a single sensor, as some of the structure is likely to lie at angles close to perpendicular to the plane. The leaf (after having been soaked in India ink for 24 hours to improve the contrast) was placed approximately perpendicular to the axis of rotation and acoustically coupled to the sensor using deionized water. The sensor plane was interrogated over a region of $20 \times 10 \mathrm{~mm}$ in $100 \mu \mathrm{m}$ step sizes. Six planes, with an angular increment of $45^{\circ}$, were imaged to give an angular aperture of $225^{\circ}$. The total size of the domain was $45 \times 45 \times 10 \mathrm{~mm}$. Fig. 4 shows maximum intensity projections of the images of the leaf phantom along three orthogonal directions. The fine structural detail of the leaf can clearly be seen to have been recovered well. The non-planar shape of the leaf can be seen in the two lateral projections, with its warped form not occupying a single plane, demonstrating the system's inherent 3D imaging capability. The darker shaded regions in the image are due to a non-uniform fluence distribution caused by parts of the leaf closer to the sensor (and to the excitation source) shading those parts of leaf further from the sensor. 

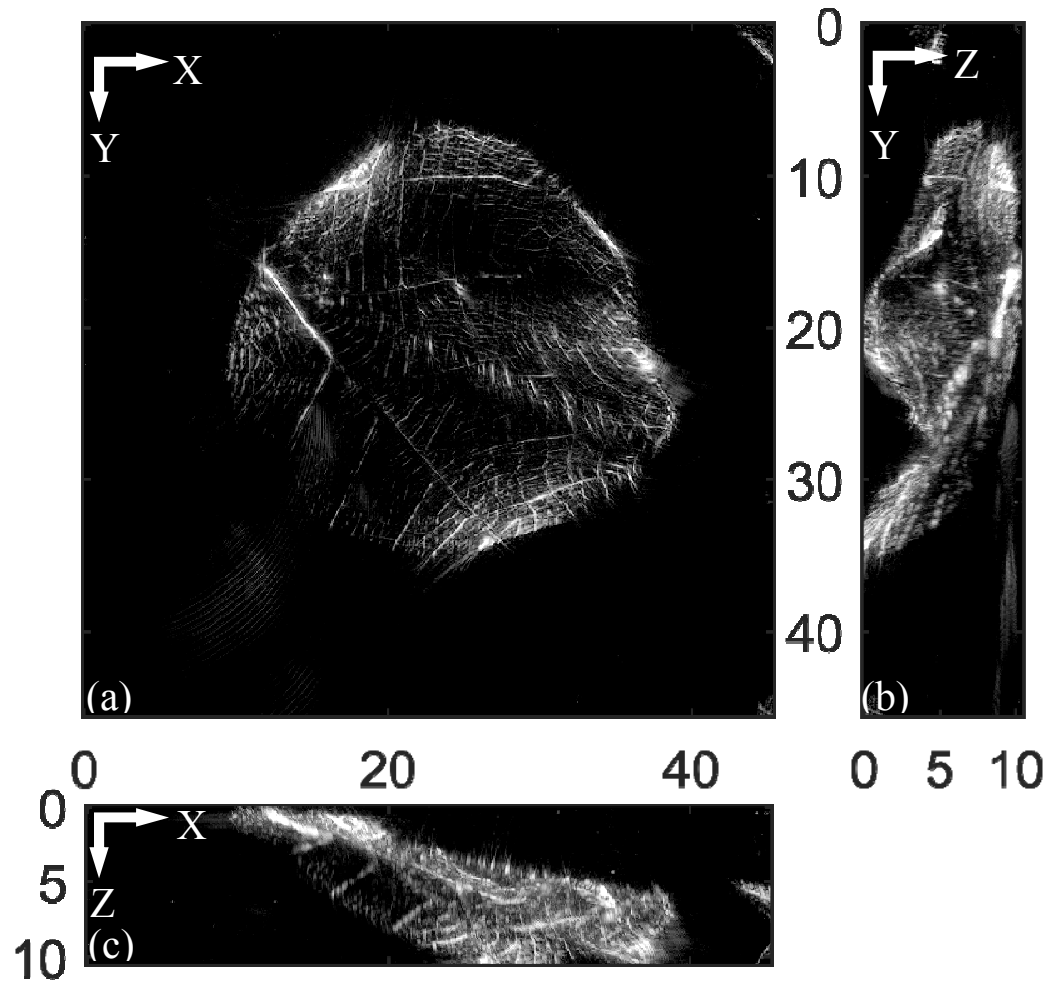

Figure 4 Reconstruction of initial pressure distribution of the leaf phantom from the multiview system. Three orientations are shown. (a) is the projection down between the sensors. (b) and (c) show the lateral projections through the sensors. Dark regions in the centre of the leaf are caused by a non-optimum fluence distribution.

\section{Tube Phantom}

The tube phantom consisted of a $580 \mu \mathrm{m}$ inner diameter tube, tied in a double coin knot. The tube was filled with India ink (diluted to $1 / 160$ ) for contrast. The phantom was acoustically coupled to the sensor using weakly scattering Intralipid $(0.05 \%)$, in order to help homogenize the fluence. The imaging parameters remained the same as for the leaf phantom. Fig. 5 shows maximum intensity projections of the reconstructed initial pressure distribution of the knot phantom along three orthogonal directions, as for the leaf phantom. The knot's structure is well recovered with no prominent artifacts. 


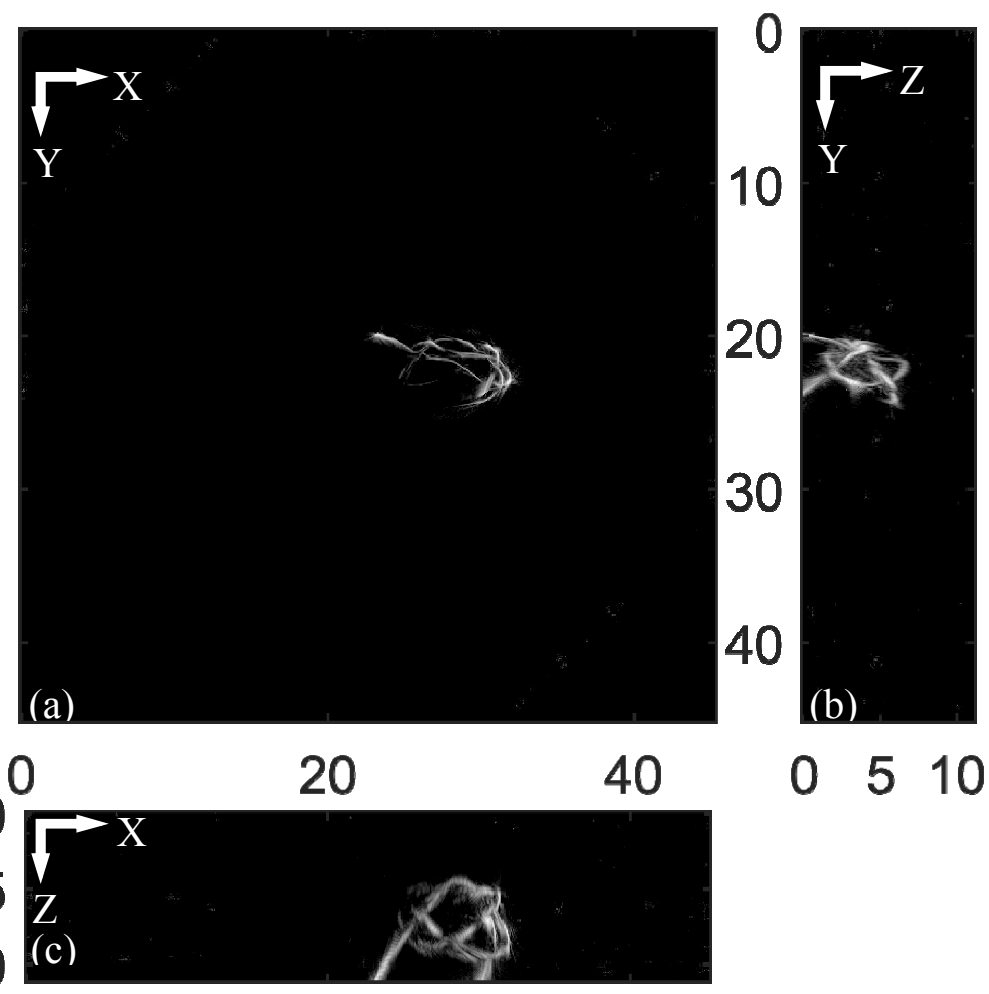

Figure 5 Reconstruction of initial pressure distribution of a knotted tube phantom from the multiview system using iterative time reversal. Three orientations are shown. (a) is the projection down between the sensors. (b) and (c) show the lateral projections through the sensors.

\section{Sub-sampling}

One advantage of the FP sensor is the possibility of sampling the acoustic field at high density with a small detector element size. This necessarily leads to a large number of measurement positions: 110000 in this study. With the current system, which measures the points sequentially, the acquisition time for all 110000 points is $\sim 2$ hours. (This is limited by the $20 \mathrm{~Hz}$ repetition rate of the excitation laser.) In order to reduce the acquisition time, the use of compressed sensing[19]-[21] with this new geometry was investigated. The full data set acquired for the knot tube phantom was subsampled spatially (but not temporally) to $1 / 6$ its original size using a random mask and then reconstructing by augmenting the iterative time reversal method used before with total variation regularization (which is conceptually similar the methods used in [21]). This would allow all six angle increments to be acquired in the time taken to acquire the full data for a single plane. (Whilst for this demonstration the sub-sampling was applied after the full data had been acquired, to reduce the acquisition time in practice, the number of measurement points would be reduced, ie. a sub-sampling scheme would be used to select the subset of points at which to collect data.) The image reconstructed from the sub-sampled data (a) and the full data set (b) can be seen in Fig. 6. It can be seen that the structure of the knot is preserved, however there is a slight degradation in the sharpness of the features in the sub-sampled image, demonstrating - for this case a trade-off between image quality and time take to acquire an image. The severity of this trade-off will, in general, depend on what is being imaged and on the regularization term used in the image reconstruction. In invivo conditions, dynamic changes of the target during data acquisition (such as motion) can significantly change this trade-off to the advantage of the sub-sampled data. 
(a)
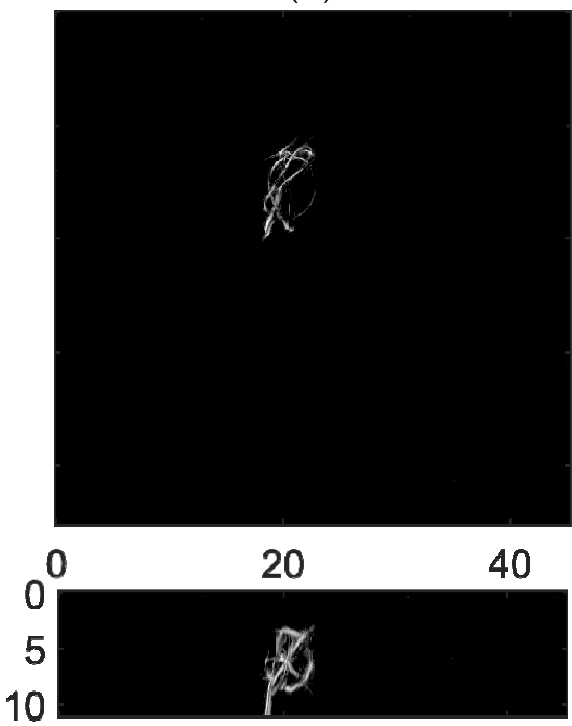

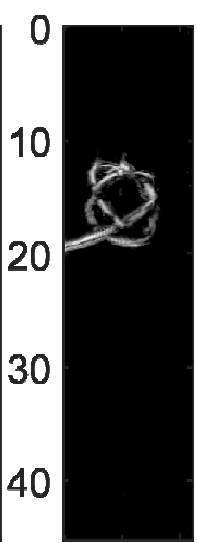

0510 (b)
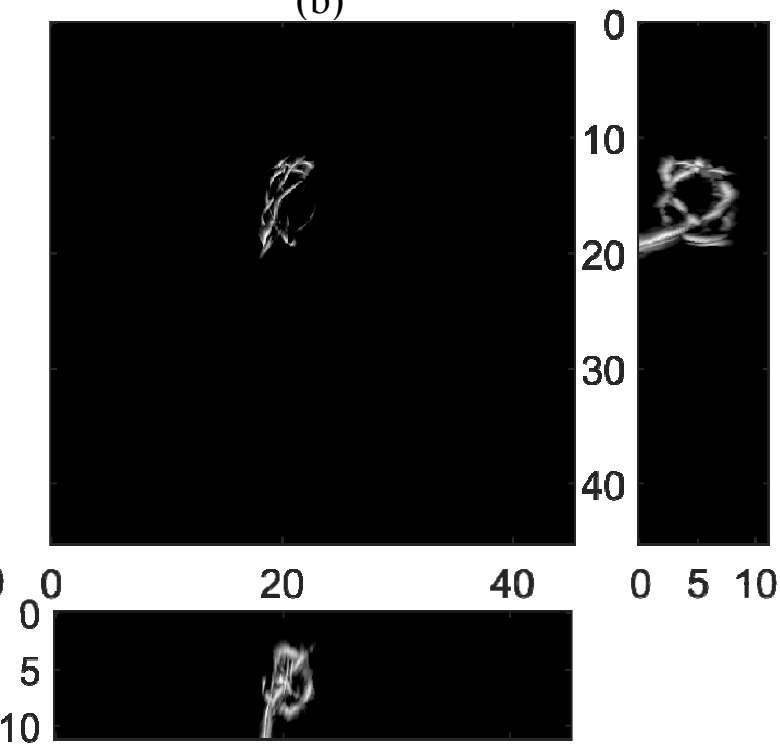

0510

Figure 6 Reconstructed knotted tube phantom with (a) full data set reconstructed and (b) sub-sampled data (1/6th original data set size) using iterative time reversal technique augmented with total variation regularisation.

\section{Conclusion and future work}

A photoacoustic tomographic imaging system based on a single FP sensor that simulated multiple planes relative to the imaged volume by rotating the target was developed. To demonstrate the system's capabilities, two phantoms were imaged: a skeletal leaf, which demonstrated the system's ability to image fine structure, and a knotted tube phantom. This system is capable of imaging a volume of $45 \times 45 \times 26 \mathrm{~mm}$ (images shown here are $45 \times 45 \times 10 \mathrm{~mm}$ in order to minimize memory requirements). In order to reduce image acquisition time a sub-sampling technique, using as many measurement points for all angles as used for a single angle in full scan mode, was investigated and shown to still produce good quality images.

Future work will include a full characterisation of the system capabilities, speeding up the data acquisition, and pre-clinical imaging demonstrations.

\section{REFERENCES}

[1] E. Zhang, J. Laufer, and P. Beard, "Backward-mode multiwavelength photoacoustic scanner using a planar Fabry-Perot polymer film ultrasound sensor for high-resolution three-dimensional imaging of biological tissues," Appl. Opt., 47, no. 4, pp. 561-577, 2008.

[2] A. P. Jathoul, J. Laufer, O. Ogunlade, B. Treeby, B. Cox, E. Zhang, P. Johnson, A. R. Pizzey, B. Philip, T. Marafioti, M. F. Lythgoe, R. B. Pedley, M. A. Pule, and P. Beard, "Deep in vivo photoacoustic imaging of mammalian tissues using a tyrosinase-based genetic reporter," Nat. Photonics, 9, no. April, pp. 239-246, 2015.

[3] Y. Xu, L. V. Wang, G. Ambartsoumian, and P. Kuchment, "Reconstructions in limited-view thermoacoustic tomography," Med. Phys., 31, no. 4, p. 724, 2004.

[4] G. Paltauf, R. Nuster, M. Haltmeier, and P. Burgholzer, "Experimental evaluation of reconstruction algorithms for limited view photoacoustic tomography with line detectors," Inverse Probl., 23, no. 6, pp. S81-S94, 2007.

[5] R. Ellwood, E. Zhang, P. Beard, and B. Cox, "Orthogonal Fabry-Pérot sensor array system for minimal-artifact photoacoustic tomography," in Proc. of SPIE, Photons Plus Ultrasound: Imaging and Sensing, 2015, 9323, p. 932312.

[6] R. Ellwood, O. Ogunlade, E. Z. Zhang, P. C. Beard, and B. T. Cox, "Orthogonal Fabry-Pérot sensors for photoacoustic tomography," in Proceedings of SPIE, 2016, 9708, p. 97082N. 
[7] R. Ellwood, O. Ogunlade, E. Zhang, P. Beard, and B. Cox, "Photoacoustic tomography using orthogonal Fabry - Pérot sensors,” J. Biomed. Opt., 22, no. 4, 2017.

[8] R. A. Kruger, W. L. Kiser, D. R. Reinecke, and G. A. Kruger, "Thermoacoustic computed tomography using a conventional linear transducer array," Med. Phys., 30, no. 5, p. 856, 2003.

[9] R. A. Kruger, W. L. Kiser, D. R. Reinecke, G. A. Kruger, and K. D. Miller, "Thermoacoustic Molecular Imaging of Small Animals,” Mol. Imaging, 2, pp. 113-123, 2003.

[10] J. Gamelin, A. Aguirre, A. Maurudis, F. Huang, D. Castillo, L. V Wang, and Q. Zhu, "Curved array photoacoustic tomographic system for small animal imaging.," J. Biomed. Opt., 13, no. 2, p. $24007,2010$.

[11]H.-P. Brecht, R. Su, M. Fronheiser, S. A. Ermilov, A. Conjusteau, and A. Oraevsky, "Whole-body three-dimensional optoacoustic tomography system for small animals," J. Biomed. Opt., 14, p. 64007, 2009.

[12]M. Oeri, W. Bost, S. Tretbar, and M. Fournelle, "Calibrated Linear Array-Driven Photoacoustic/Ultrasound Tomography," Ultrasound Med. Biol., 42, 11 pp. 1-11, 2016.

[13] Y. Xu and L. Wang, "Time Reversal and Its Application to Tomography with Diffracting Sources," Phys. Rev. Lett., 92, no. 3, pp. 3-6, 2004.

[14]P. Burgholzer, G. Matt, M. Haltmeier, and G. Paltauf, "Exact and approximative imaging methods for photoacoustic tomography using an arbitrary detection surface,” Phys. Rev. E, 75, no. 4, pp. 1-10, 2007.

[15]A. Myronenko and X. Song, "Point set registration: coherent point drift," IEEE Trans Pattern Anal Mach Intell, 32, no. 12, pp. 2262-2275, 2010.

[16] G. Paltauf, J. A. Viator, S. A. Prahl, and S. L. Jacques, "Iterative reconstruction algorithm for optoacoustic imaging," J. Acoust. Soc. Am., 112, no. 4, p. 1536, 2002.

[17] P. Stefanov and G. Uhlmann, "Thermoacoustic tomography with variable sound speed," Inverse Probl., 25, no. 7, p. 75011, 2009.

[18]B. E. Treeby and B. T. Cox, "k-Wave: MATLAB toolbox for the simulation and reconstruction of photoacoustic wave fields," J. Biomed. Opt., 15, no. 2, p. 21314, 2010.

[19] J. Provost and F. Lesage, "The application of compressed sensing for photo-acoustic tomography.," IEEE Trans. Med. Imaging, 28, no. 4, pp. 585-94, 2009.

[20]Z. Guo, C. Li, L. Song, and L. V Wang, "Compressed sensing in photoacoustic tomography in vivo.," J. Biomed. Opt., 15, no. 2, p. 21311, 2011.

[21] S. R. Arridge, P. Beard, M. M. Betcke, B. Cox, N. Huynh, F. Lucka, O. Ogunlade, and E. Zhang, "Accelerated High-Resolution Photoacoustic Tomography via Compressed Sensing," Phys Med Biol, 61, pp. 8908-8940, 2016. 